\title{
Editorial zur Ausgabe „Fall verstehen: Diagnostik und Psychotherapie"
}

\author{
Doris Beneder · Rudolf Agner
}

(C) Springer-Verlag GmbH Austria, part of Springer Nature 2020

Mitten im Begutachtungsprozess dieses Doppelheftes hat uns die „Corona-Krise“ getroffen. Eine Autorin konnte wegen ihrer Erkrankung den Beitrag nicht fertig stellen, da sie gesundheitlich über einen längeren Zeitraum sehr belastet war, und wir sind froh, dass sie wieder gesund ist. Gutachter*innen und $\mathrm{Au}-$ tor*innen waren gefordert, viele mussten ihre Lehrgänge auf virtuelle Lernformen umstellen, Krisenhotlines wurden eingerichtet, Klient*innen mussten betreut werden u.v.m. Wir bedanken uns bei allen Mitwirkenden, die trotz unterschiedlichster Belastungen ihre Aufgaben für das Psychotherapie Forum weiterverfolgt haben. Unser Dank gilt auch den Verlagsmitarbeiter*innen, die sich flexibel auf kurzfristige Veränderungen im Produktionsprozess eingestellt haben. Herzlichen Dank an alle!

In dieser Ausgabe beschäftigt uns die Frage nach einer das Fallverständnis unterstützenden und dem psychotherapeutischen Prozess angemessenen Diagnostik. Weiterentwicklungen und aktuelle Forschungsergebnisse zu dieser spezifisch psychotherapeutischen Herangehensweise entlang der vier großen Orientierungen - tiefenpsychologisch, humanistisch, verhaltenstherapeutisch und systemisch - werden vorgestellt.

Trotz vieler Unterschiede weisen die Beiträge als gemeinsames Verständnis das Zusammenwirken von Erkennen und Verändern, von Diagnostik und Therapie, über den gesamten Therapieprozess auf. Darin zeigt sich der Unterschied der entwicklungsorientierten Diagnostik in der Psychotherapie im Vergleich zur Statusdiagnostik in anderen, eher am medizinischen Modell orientierten Gesundheitsberufen. Gleichzeitig

\section{Beneder $(\bowtie)$}

Psychotherapie Forum, Österreichischer Bundesverband für Psychotherapie (ÖBVP), Löwengasse 3, 1030 Wien, Österreich

oebvp.psychotherapieforum@psychotherapie.at wird eine, bereits in der Diagnostik-Leitlinie (2004) angelegte, pragmatische Anpassung an die Klassifikationssysteme ICD und DSM offensichtlich, auf die in der Mehrheit der Beiträge Bezug genommen wird.

Einige Beiträge stellen erste Ergebnisse (noch) nicht publizierter Studien vor, in denen die Frage des Fallverständnisses und des damit verbundenen Menschenbildes explizit aufgegriffen wird. An diesen Beispielen wird deutlich, dass sich der oft mühsame Weg der methodenübergreifenden Annäherung als fruchtbar und praxisrelevant erweist.

\section{Die Beiträge im Einzelnen}

Die Erstellung von klinischen Einzelfallstudien ist Teil nahezu jeder psychotherapeutischen Ausbildung in Österreich und dient als Nachweis von Wissen, Reflexionsfähigkeit und methodisch-praktischen Fertigkeiten der Kandidaten*innen. Die Autorinnen Ela Neidhart und Henriette Löfler-Staska befragten 34 Ausbildungseinrichtungen zu deren Vorgangsweisen und Qualitätsstandards. Sie erkennen Einzelfallstudien als probates wissenschaftliches Forschungsmittel, wenn bestimmte Mindeststandards umgesetzt werden, weshalb sie für die Erarbeitung einer Methoden-übergreifenden Leitlinie für Einzelfallstudien plädieren.

Anhand von zwei Fallbeispielen aus einer Pilotstudie, die der Verein Dialog in Kooperation mit dem Institut für Erziehungshilfe Wien durchführt, werden der diagnostische Prozess der Studie, sowie erste Veränderungen der Mentalisierungsfähigkeit durch Gruppeninterventionen bei fremduntergebrachten Kindern und deren Bezugsbetreuer*innen exemplarisch vorgestellt. Beachtenswert ist die Einbeziehung der Bezugsbetreuer*innen und das differenzierte Design. Die Autorinnen Nadja Springer und Brigitte Lueger-Schuster gewähren einen ersten Einblick in 
die Studie, sodass man mit Spannung die vollständige Publikation erwartet.

Ein Autor*innen Team der Arbeitsgemeinschaft für Verhaltensmodifikation (AVM) unter der Leitung von Horst Mittmansgruber beschreibt am Beispiel einer 34-jährigen depressiven Patientin die Entwicklung einer individualisierten Fallkonzeption. Das klassische Diathese-Stress Modell wird in der vertikalen Verhaltensanalyse um biografisch nachvollziehbare Grundannahmen ergänzt, wobei die gemeinsame Erarbeitung eines hypothetischen Bedingungsmodells, das im Prozess immer detaillierter spezifiziert wird, auch für die Gestaltung der therapeutischen Beziehung aufschlussreich ist.

Astrid Görtz stellt am Beispiel einer Patientin mit einer generalisierten Angststörung das Zusammenwirken von phänomenologisch-hermeneutischer Diagnose, therapeutischer Intervention und Evaluation dar, wobei das Modell der Personalen Existenzanalyse als Rahmenmodell dient.

Elisabeth Wagner fokussiert in ihrem Beitrag auf ein synergetisches Fallverständnis, das die Wechselwirkungen von Kontextbedingungen und intrapsychischen Prozessen gleichermaßen berücksichtigt. Individuelle Musterbildungen können in einem gemeinsamen Such- und Erkundungsprozesse entdeckt und schließlich verändert werden.

Petra Klampfl und Markus Hochgerner diskutieren die Möglichkeit der Integration der psychodynamisch konzipierten Operationalisierten Psychischen Diagnostik (OPD) in Konzepte der Integrativen Gestalt- therapie am Beispiel einer Patientin mit schwerem strukturellen Defizit.

Am Beispiel einer 15-jährigen Jugendlichen beschreibt Marie-Luise Brückl den entwicklungsfördernden Blick auf das Fallverständnis aus Personzentrierter Perspektive. Der Prozess von Verstehen und Verstanden-Werden unterstützte die Jugendliche den inneren Bezugsrahmen zu verändern und damit ihr Selbstkonzept zu stabilisieren.

In drei ausführlich vorgestellten Fallvignetten beschreiben Susanne Ohmann, Bibiana Schuch und Christian Popow den diagnostisch-therapeutischen Prozess und die abschließende Evaluation in der kognitiven Verhaltenstherapie. Für den Therapieerfolg ist die Einbindung des familiären und schulischen Umfeldes in den gesamten Prozess von zentraler Bedeutung.

Die Originalarbeiten werden durch zum Themenschwerpunkt passende Buchrezensionen ergänzt.

Doris Beneder \& Rudolf Agner

Verantwortliche Editorin in Chief \& Assistant Editor für die Doppelausgabe Fall verstehen: Diagnostik und Psychotherapie

Interessenkonflikt D. Beneder und R. Agner geben an, dass kein Interessenkonflikt besteht.

Hinweis des Verlags Der Verlag bleibt in Hinblick auf geografische Zuordnungen und Gebietsbezeichnungen in veröffentlichten Karten und Institutsadressen neutral. 